\title{
Endogenous transmembrane protein UT2 inhibits pSTAT3 and suppresses hematological malignancy
}

\author{
Dongjun Lee, ${ }^{1,2,3,4}$ Ying-Hua Wang, ${ }^{1,2,3,4}$ Demetrios Kalaitzidis, ${ }^{1,2,3,4}$ Janani Ramachandran, ${ }^{2}$ Homare Eda, ${ }^{2}$ David B. Sykes, ${ }^{1,2,3,4}$ \\ Noopur Raje, ${ }^{2}$ and David T. Scadden ${ }^{1,2,3,4}$
}

'Center for Regenerative Medicine and ${ }^{2}$ Cancer Center, Massachusetts General Hospital, Boston, Massachusetts, USA. ${ }^{3}$ Department of Stem Cell and Regenerative Biology and ${ }^{4}$ Harvard Stem Cell Institute, Harvard University, Cambridge, Massachusetts, USA.

\begin{abstract}
Regulation of STAT3 activation is critical for normal and malignant hematopoietic cell proliferation. Here, we have reported that the endogenous transmembrane protein upstream-of-mTORC2 (UT2) negatively regulates activation of STAT3. Specifically, we determined that UT2 interacts directly with GP130 and inhibits phosphorylation of STAT3 on tyrosine 705 $\left(\mathrm{STAT}^{\mathrm{Y705}}\right.$ ). This reduces cytokine signaling including IL6 that is implicated in multiple myeloma and other hematopoietic malignancies. Modulation of UT2 resulted in inverse effects on animal survival in myeloma models. Samples from multiple myeloma patients also revealed a decreased copy number of UT2 and decreased expression of UT2 in genomic and transcriptomic analyses, respectively. Together, these studies identify a transmembrane protein that functions to negatively regulate cytokine signaling through GP130 and $\mathrm{PSTAT} 3^{\mathrm{V} 705}$ and is molecularly and mechanistically distinct from the suppressors of cytokine signaling (SOCS) family of genes. Moreover, this work provides evidence that perturbations of this activation-dampening molecule participate in hematologic malignancies and may serve as a key determinant of multiple myeloma pathophysiology. UT2 is a negative regulator shared across STAT3 and mTORC2 signaling cascades, functioning as a tumor suppressor in hematologic malignancies driven by those pathways.
\end{abstract}

\section{Introduction}

We identified UT2 as a transmembrane molecule altered in leukemic cells that emerged from an animal model with modifications in specific BM stromal cells (1). Hypothesizing that the genes altered in the malignant cells that emerge from this niche-induced oncogenesis model might reflect how an abnormal microenvironment leads to cancer, we focused on those genes encoding transmembrane molecules. UT2 was one such molecule. UT2 interacts directly with RICTOR and thereby inhibits mTOR kinase activity in the RICTOR-containing mTORC2 complex (2). The molecule was therefore named upstream-of-mTORC2 (UT2). Increased expression of UT2 prolonged survival in NOTCH-induced T cell acute lymphoblastic leukemia (T-ALL) mouse models (2). However, increasing UT2 had effects beyond that of RICTOR deletion, and therefore, we explored other roles for UT2. Specifically, we observed that UT2 altered hematopoietic cell growth in ways that could not be explained by its effects on mTORC2, made evident by differing phenotypes of UT2 overexpression and RICTOR deletion. We therefore sought to define if UT2 interacted with other signaling pathways relevant for hematopoietic growth. Here, we report that UT2 inhibits STAT3 signaling by directly binding to GP130.

STAT3 participates in malignant transformation, tumor cell survival, invasion, and metastasis (3). STAT3 activation has been detected in carcinomas and hematologic malignancies (4-7), including multiple myeloma (MM) and leukemia $(8,9)$. Other studies have

Conflict of interest: The authors have declared that no conflict of interest exists. Submitted: September 10, 2015; Accepted: January 14, 2016.

Reference information: / Clin Invest. 2016;126(4):1300-1310. doi:10.1172/JCI84620. demonstrated that activation of STAT3, downstream of GP130, is critical for the regulation of hematopoietic cell survival and proliferation (10-14). STAT3 is activated by multiple paths, including the IL6 family of cytokines and Janus-activated kinase (JAK) phosphorylation of the IL6 receptor (IL6R) and the subsequent recruitment and phosphorylation of STAT3 on tyrosine 705 (STAT3 ${ }^{\mathrm{Y} 705}$ ) (15). In the IL6-IL6R cascade, this IL6-IL6R complex induces the homodimerization of two GP130 molecules $(16,17)$, leading to intracellular signaling events, including tyrosine phosphorylation of GP130, which is critical for the activation of STAT3 $(18,19)$.

JAK-STAT activation is negatively regulated by a family of genes known as suppressors of cytokine signaling (SOCS) (20). These molecules are a family of intracellular proteins and contain shared Src homology 2 (SH2) domain and molecular SOCS box motifs. Among them, SOCS3 binds and competes with SHP2 in binding GP130 and, as it does so, inhibits IL6 signal transduction $(21,22)$. SOCS3 expression is induced by pSTAT3 to serve as a negative feedback loop for cytokine signaling (23-26); the negative regulation of STAT3 imposed by UT2 is distinct from this and other previously defined STAT3 inhibitors as it works upstream of STAT3 activation.

The association of STAT3 activation with cancer is well established and is particularly well defined in myeloma (27). For example, constitutive activation of GP130/JAK/STAT3 signal transduction was sufficient to induce MM in a murine model in vivo (27). Furthermore, IL6 $(7,10)$ and constitutive STAT3 activation has been shown in human myeloma cells (7). Activation of STAT3 and target genes occurs in human MM patients (27), and IL6-induced pSTAT3 is a prognostic biomarker for improved survival in MM patients (28). Furthermore, blocking IL6-IL6R and/or inhibiting 

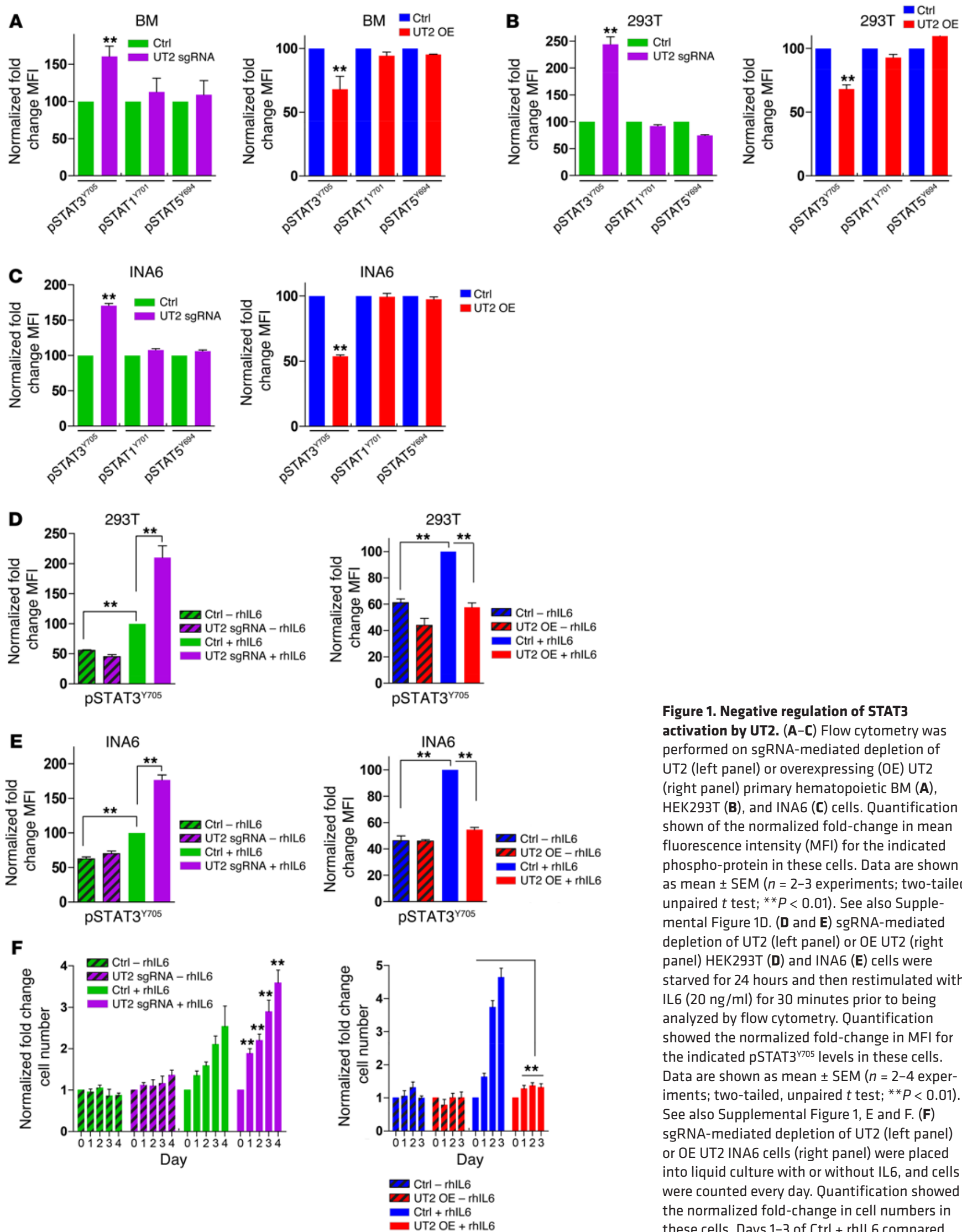

Figure 1. Negative regulation of STAT3 activation by UT2. (A-C) Flow cytometry was performed on sgRNA-mediated depletion of UT2 (left panel) or overexpressing (OE) UT2 (right panel) primary hematopoietic BM (A), HEK293T (B), and INA6 (C) cells. Quantification shown of the normalized fold-change in mean fluorescence intensity (MFI) for the indicated phospho-protein in these cells. Data are shown as mean \pm SEM ( $n=2-3$ experiments; two-tailed, unpaired $t$ test; ${ }^{* *} P<0.01$ ). See also Supplemental Figure 1D. (D and E) sgRNA-mediated depletion of UT2 (left panel) or OE UT2 (right panel) HEK293T (D) and INA6 (E) cells were starved for 24 hours and then restimulated with IL6 $(20 \mathrm{ng} / \mathrm{ml})$ for 30 minutes prior to being analyzed by flow cytometry. Quantification showed the normalized fold-change in MFI for the indicated pSTAT3 $3^{\mathrm{V705}}$ levels in these cells. Data are shown as mean \pm SEM $(n=2-4$ experiments; two-tailed, unpaired $t$ test; ${ }^{* *} P<0.01$ ). See also Supplemental Figure 1, E and F. (F) sgRNA-mediated depletion of UT2 (left panel) or OE UT2 INA6 cells (right panel) were placed into liquid culture with or without IL6, and cells were counted every day. Quantification showed the normalized fold-change in cell numbers in these cells. Days 1-3 of Ctrl + rhIL6 compared with corresponding days of UT2 OE + rhIL6. Data are shown as mean \pm SEM ( $n=2$ experiments; two-tailed, unpaired $t$ test; $\left.{ }^{* *} P<0.01\right)$. - rhIL6, rhIL6 withdrawal. 
STAT3 activation was shown to arrest growth and increase apoptosis in myeloma cell lines (29). Inhibition of STAT3 was shown to have anticancer activity in vitro and in animal models (30-34). Furthermore, direct inhibition of GP130 attenuates STAT3 activation and inhibits some cancer cells (9). Inhibitors of STAT3 activation have been shown to be active in MM, and clinical trials using these inhibitors are being conducted (35). Therefore, the IL6-STAT3 axis is a key participant in myeloma biology, and defining novel methods for inhibiting that pathway are of clinical relevance.

Here, we define UT2 as an endogenous negative regulator of the IL6-STAT3 signaling axis. Because of the connection of IL6STAT3 signaling to MM, we examined UT2 in the context of that disease and demonstrate that myeloma cell growth in vitro and in vivo is inhibited by UT2. Furthermore, UT2 loss or suppression is evident in patient samples, and patients whose tumors express less of the inhibitor UT2 have a worse clinical outcome. Therefore, these data add a unique, transmembrane suppressor of cytokine signaling highly relevant for human disease. It is not a member of the SOCS family by either molecular structure or function, but it serves a similar role in constraining cell activation. UT2 is a distinctive molecular inhibitor of both the STAT3 and mTORC2 signaling pathways, and its transmembrane location makes it readily accessible for pharmacologic targeting.

\section{Results}

UT2 is a negative regulator of STAT3 activation. We edited UT2 using the CRISPR-associated nuclease Cas9 genome-editing system (refs. 36, 37, and Supplemental Figure 1, A and B; supplemental material available online with this article; doi:10.1172/ JCI84620DS1). Genome targeting of UT2 increased the level of $\mathrm{pAKT}^{\mathrm{S} 473}$ in association with depleted UT2 protein levels (Supplemental Figure 1A), consistent with our prior findings (2). Unexpectedly, we also noted that STAT3 activation was influenced by the levels of UT2 in primary mouse hematopoietic cells (Figure 1A and Supplemental Figure 1, A and D), with UT2 single guide RNA (sgRNA) deletion increasing pSTAT3 ${ }^{\mathrm{Y} 705}$, the critical site for STAT3 activity, but not pSTAT1 ${ }^{\mathrm{Y} 701}$ or pSTAT5 ${ }^{\mathrm{Y} 694}$. Conversely, UT2 overexpression decreased pSTAT3 $3^{\mathrm{Y} 705}$ but not pSTAT $^{\mathrm{Y} 701}$ or pSTAT5 $^{\mathrm{Y} 694}$. Since HEK293T cells better enable biochemical studies, utilize STAT3 and AKT signaling, and endogenously express GP130, IL6R, and UT2 (38-44), we also examined them and found that UT2 altered STAT3 phosphorylation in a similar manner (Figure $1 \mathrm{~B}$ and Supplemental Figure 1D). Because the IL6-IL6R/GP130STAT3 cascade is implicated in myeloma, we explored the functional role of UT2 in myeloma cells (Figure 1C and Supplemental Figure 1, A and D). Interestingly, sgRNA deletion of UT2 increased pSTAT3 ${ }^{\mathrm{Y} 705}$ levels in IL6-supported INA6 myeloma cells. Moreover, we examined expression of the bona fide STAT3 target gene SOCS3 (45) and found that it was increased in sgRNA deletion of UT2 primary BM, HEK293T, and INA6 cells (Supplemental Figure 1C).

Next, we examined whether UT2 altered the phosphorylation levels of pSTAT3 ${ }^{\mathrm{Y} 705}$ in response to IL6 $(15,18)$. Inhibiting UT2 by sgRNA led to an increase in IL6-induced pSTAT3 ${ }^{\mathrm{Y705}}$ in HEK293T and INA6 cells (Figure 1, D and E, left panels, and Supplemental Figure 1, E-G). Conversely, phosphorylation of STAT3 was attenuated in HEK293T and INA6 cells expressing UT2 in response to IL6 (Figure 1, D and E, right panels, and Supplemental Figure 1, E, F, and H).
We assessed cell growth from the IL6-dependent INA6 myeloma cells, with or without IL6 treatment (Figure 1F). sgRNA deletion of UT2 significantly increased INA6 cellular growth in the presence of IL6 (Figure 1F, left panel). Furthermore, INA6 cells expressing UT2 had markedly reduced proliferation compared with control cells (Figure 1F, right panel). These data demonstrate the ability of UT2 modulation to alter myeloma cell growth.

UT2 interacts with GP130. Given the impact of UT2 on the GP130-STAT3 axis, we assessed whether UT2 physically interacts with GP130. We conducted immunoprecipitation (IP) experiments on HEK293T cells expressing full-length UT2 or UT2 with deletion of the cytoplasmic portion of the molecule (UT2 $\Delta \mathrm{C}$ ) (Figure 2A). Anti-FLAG monoclonal antibody used for IP demonstrated coprecipitation of STAT3, IL6R, and GP130 by Western blot. We therefore explored the endogenous interaction further in HEK293T and INA6 cells using antibodies against UT2 for IP, and we could detect coimmunoprecipitated GP130, STAT3, and IL6R (Figure 2B). These data are consistent with an interaction of UT2 with the IL6R/GP130/STAT3 complex.

We next tested whether UT2 interaction with GP130 had a meaningful impact on the function of STAT3. Using GP130 IP of HEK293T cells expressing UT2 (Figure 2C) or sgRNA-mediated depletion of UT2 (Figure 2D), we could demonstrate different levels of GP130 compared with controls. IP experiments on HEK293T cells expressing UT2 using anti-GP130 antibody brought down less GP130 (Figure 2C), suggesting that UT2 affects GP130 homomolecular interaction. Conversely, inhibiting UT2 by sgRNA led to increased GP130 levels brought down after conducting GP130 IP (Figure 2D). To further verify a direct interaction between UT2 and GP130, in vitro translated (IVT) proteins were generated, and IP experiments were conducted (Figure 2E). IP of UT2 and GP130 demonstrated co-IP indicative of a direct interaction in vitro (Figure $2 \mathrm{E}$ ). However, conducting the same experiment of IVT UT2 with IVT STAT3 and IL6R failed to demonstrate any co-IP of UT2 (Supplemental Figure 2). The presence of UT2 led to decreased GP130-GP130 interaction in vitro (Figure 2E). Cumulatively, these data indicate that UT2 directly binds GP130 and affects its ability to interact with other GP130 molecules.

UT2 expression in myeloma cells. To test whether UT2 affects human hematological malignancies broadly, we first conducted in silico screening of the comparative genomic hybridization (CGH) array database at the Broad-Novartis Cancer Cell Line Encyclopedia (https://www.broadinstitute.org/ccle/home) and Oncomine (https://www.oncomine.org) websites (ref. 46 and Figure 3A). These studies enabled us to explore UT2 copy number in hematological cancer cell lines (Figure 3A) and UT2 expression in patient samples (refs. 47-54 and Supplemental Figure 3A). Interestingly, UT2 copy number and expression are profoundly decreased in hematologic malignancies, including leukemia, MM, and kidney cancers (Figure 3A and Supplemental Figure 3A). Thus, we sought to determine whether UT2 affects human MM patients; we conducted in silico screening of the CGH array $(55,56)$ and microarray database (55-59) through the Oncomine website to explore the UT2 copy number and expression from human MM patients in genomic and transcriptomic analyses, respectively (Figure 3, B and $\mathrm{C}$ ). We found that both UT2 copy number and expression are decreased in MM patient samples (Figure 3, B and C). 
A

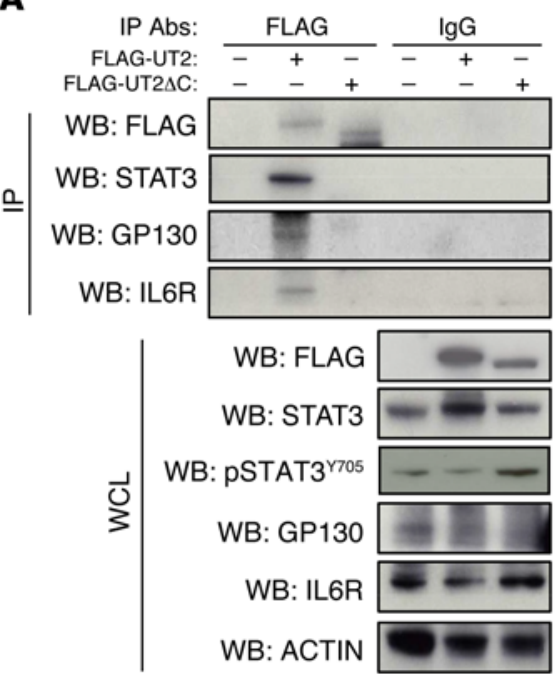

B

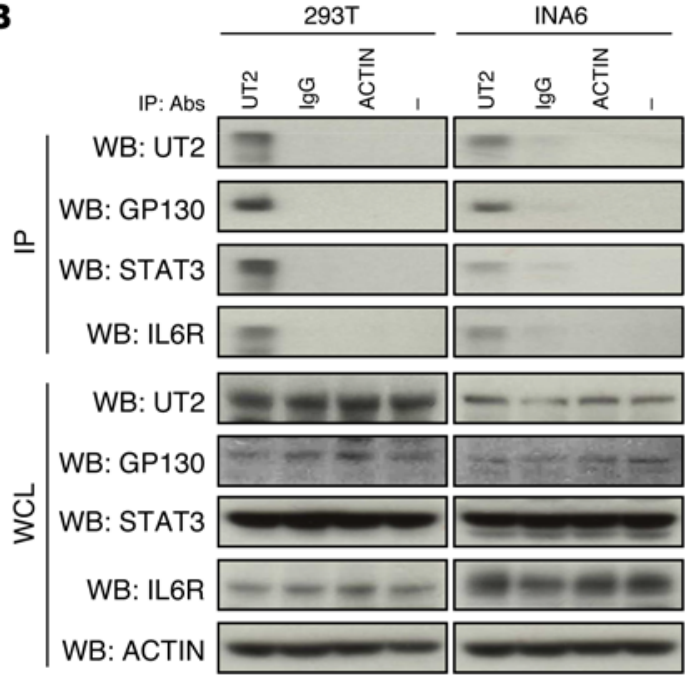

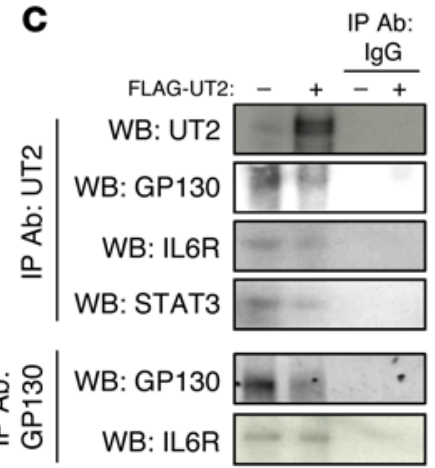
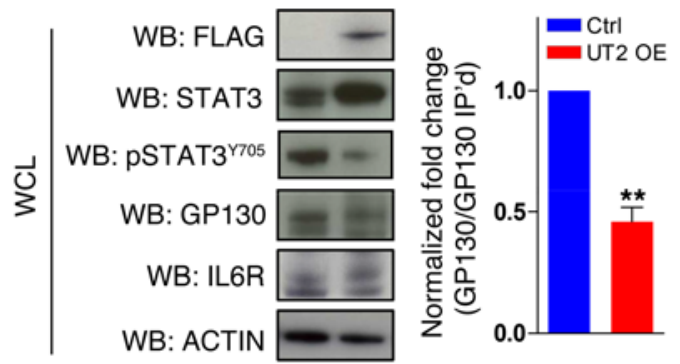

D
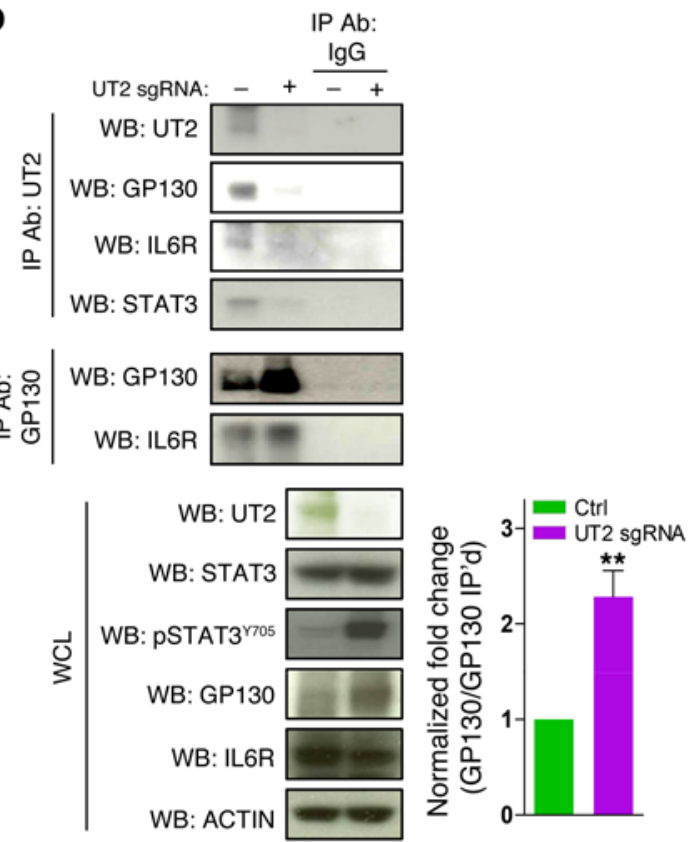

$\mathbf{E}$
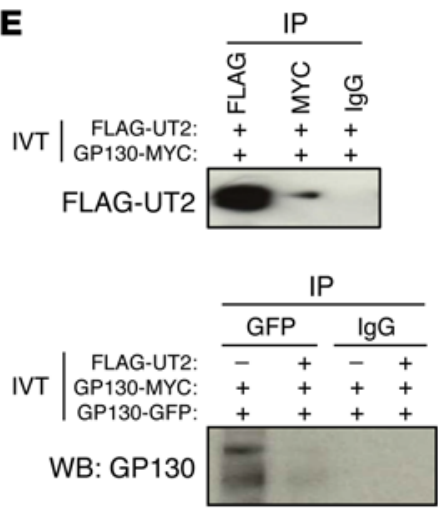

WB: FLAG-UT2

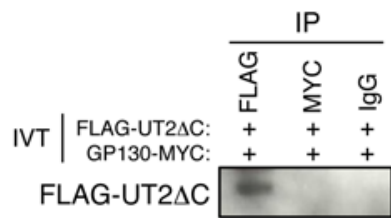

FLAG-UT2 $\Delta C$

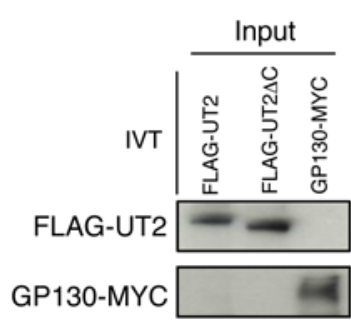

Figure 2. UT2 interacts with GP130. (A) HEK293T cells expressing either FLAG-UT2 full length or FLAG-UT2 $\Delta$ C mutant were lysed and subjected to IP using an antibody directed against FLAG and $\operatorname{IgG}$, respectively. The resulting precipitates and the corresponding whole-cell lysates (WCL) were subjected to Western blot analysis using the indicated antibodies. (B) UT2 IPs were prepared from HEK293T (left panel) and INA6 (right panel) cells and subjected to Western blot analysis using the indicated antibodies. (C and D) UT2 or GP130 were immunoprecipitated from overexpressing (OE) UT2 (C) or sgRNA-mediated depletion of UT2 (D) HEK293T cells and subjected to Western blot analysis using the indicated antibodies. Infrared Western blotting (Odyssey system) was used for quantification. The fold-change in GP130 was normalized to GP130 levels in control cells. Data are shown as mean \pm SEM ( $n=4$ experiments; two-tailed, unpaired $t$ test; ${ }^{*} P<0.01$ ). (E) IVT either FLAG-UT2 full length or FLAG-UT2 $\Delta C$ mutant proteins were subjected to IP using the indicated antibodies in the presence of in vitro-translated GP130 proteins. WB, Western blot. 
A Broad-Novartis cancer cell line encyclopedia $(n=338)$
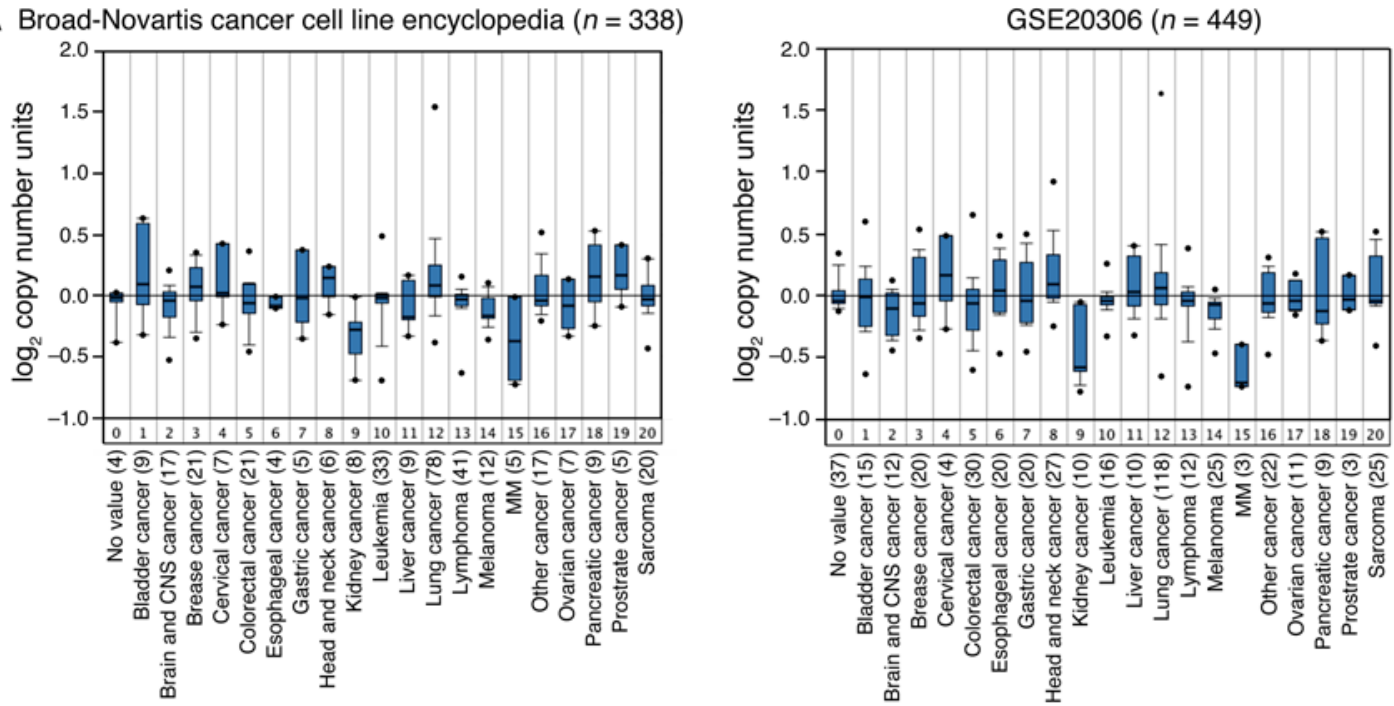

B
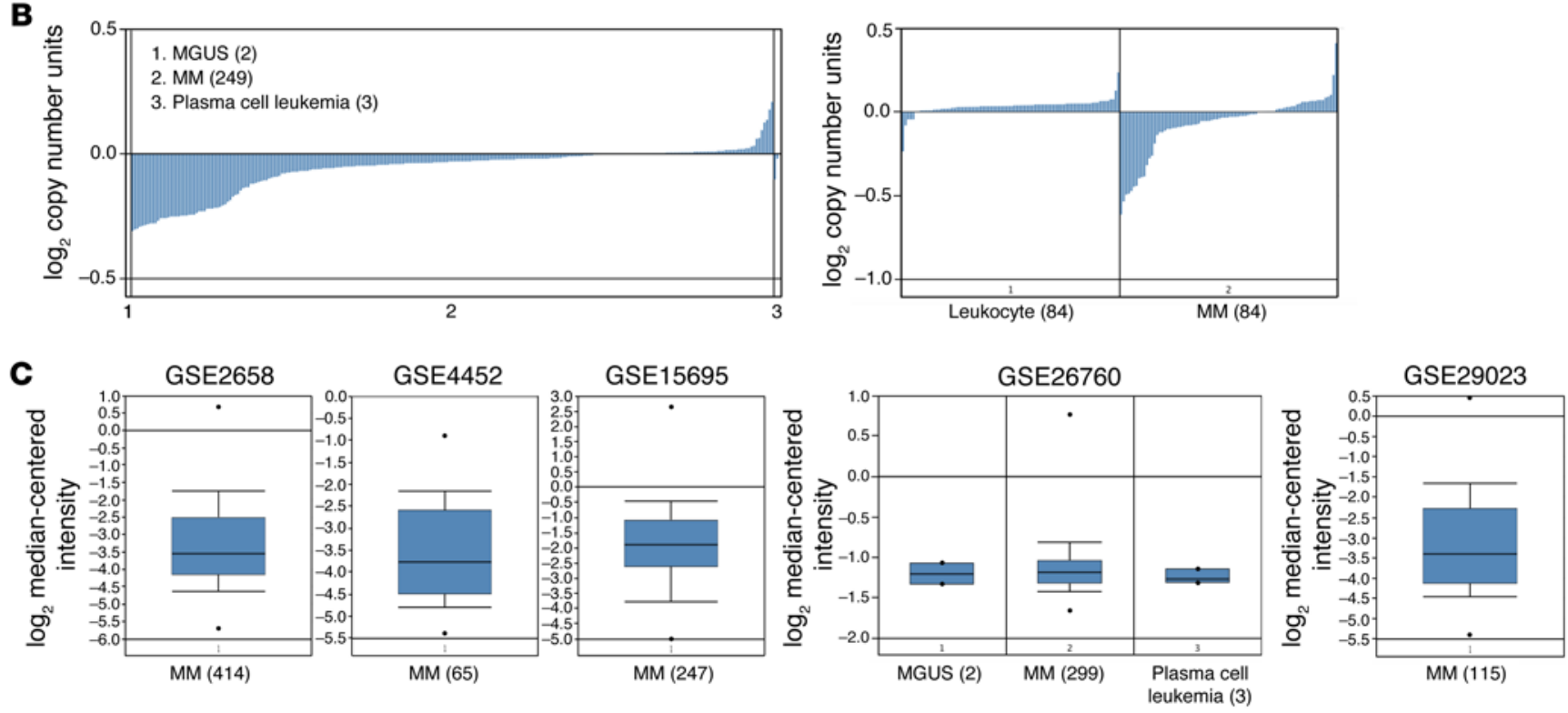

D
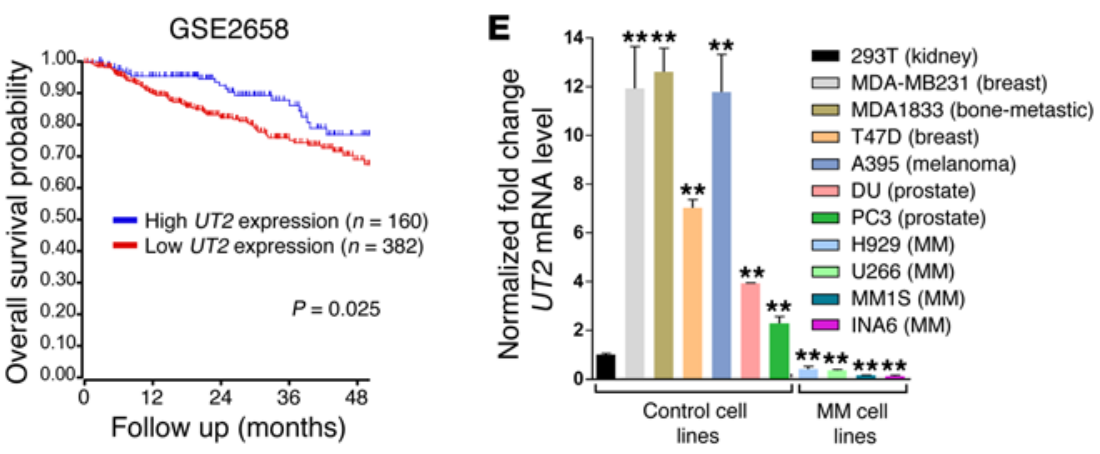

G

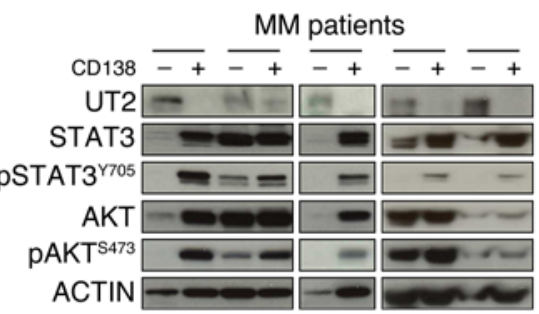

$\mathbf{F}$
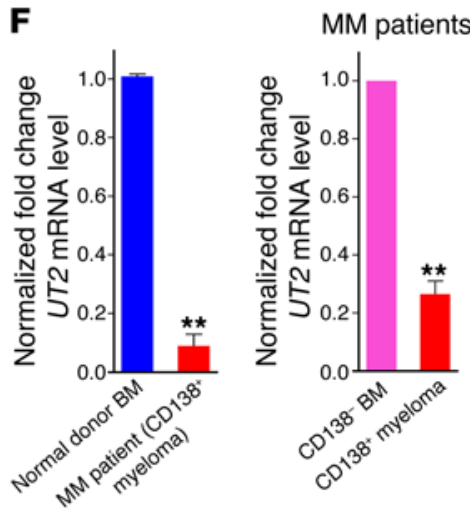
Figure 3. UT2 expression in myeloma cells. (A) Copy number of UT2 in solid tumors and hematological cancer cell lines (Broad-Novartis Cancer Cell Line Encyclopedia [ $n=338$; left panel] and Oncomine database corresponds to GSE20306 data [ $n=449$ ] derived from ref. 46 [right panel]). Numbers in parentheses are $n$ values. (B) Copy number of UT2 in primary MM patients were assessed using human genome CCH microarray data obtained from the Oncomine database. Left panel corresponds to GSE26849 data (MGUS [ $n=2]$, MM [ $n=249$ ], and plasma cell leukemia $[n=10]$ ) from ref. 56 , and right panel corresponds to GSE15695 data (normal donor $[n=84]$ and MM $[n=84]$ ) from ref. 55. (C) UT2 expression from primary MM patients were assessed using human genome $\mathrm{CCH}$ microarray data obtained from the Oncomine database. [ panel corresponds to data from refs. 55-59 (CSE2658 data [ $n=414]$, CSE4452 data [ $n=65$ ], GSE15695 data [ $n=247$ ], GSE26760 data [MGUS $(n=2)$, MM ( $n=299$ ), and plasma cell leukemia $(n=3)]$, and GSE29023 data [ $n=115]$ ). (D) Survival curve relative to UT2 expression (high expression $[n=160]$ and low expression $[n=382])$ in individuals affected by myeloma and based on CSE2658 (58) (log-rank test). (E) Quantitative PCR (qPCR) analyses of UT2 expression in different human control cancer cells and myeloma cells ( $n=3$ experiments; two-tailed, unpaired $t$ test; ${ }^{* *} P<0.01$ ). (F and $\left.\mathbf{G}\right)$ qPCR and Western blots analyses of UT2 expression from primary MM patients. CD138+ myeloma cells and CD138- BM cells were isolated from primary MM patient BM cells, and qPCR analysis (F) (normal donor BM cells [left panel] and CD138- BM fractions [right panel]) and Western blot analysis (G) were performed, respectively. See also Supplemental Figure 3B. Data are shown as mean \pm SEM $\left(n=5-6\right.$; two-tailed, unpaired $t$ test; $\left.{ }^{*} P<0.01\right)$.

We next explored the overall survival of MM patients based on relative levels of UT2 expression (ref. 58 and Figure 3D). Individuals with low UT2 expression (expected to have less inhibition of pSTAT3) had significantly shorter survival than those with high expression. Moreover, to validate our findings in myeloma cells, we tested the UT2 expression in various myeloma cell lines and observed a consistent, significant reduction of UT2 expression in myeloma cells compared with other cell lines (Figure 3E).

Furthermore, to investigate whether UT2 expression is altered in $\mathrm{MM}$ patients, we purified the $\mathrm{CD} 138^{+}$fraction in primary $\mathrm{BM}$ cells from primary MM patients (Supplemental Figure 3B) and tested UT2 expression (Figure 3, F and G). We noted reduced UT2 expression in $\mathrm{MM}$ BM cells in comparison with normal donor BM cells (Figure 3F, left panel). In MM patient BM cells, UT2 expression is lower in $\mathrm{CD} 138^{+}$myeloma cells than in the CD138- fraction (Figure 3, F, right panel, and G). Moreover, pSTAT3 and pAKT levels are higher in $\mathrm{CD}_{138^{+}}$myeloma cells than in the CD138- fraction. Activation of STAT3 and AKT occurs in human MM patients $(27,28,56,59)$. These data demonstrate an inverse correlation between UT2 levels and STAT3/AKT phosphorylation in MM patients, as would be predicted from our proposed relationship between these molecules. Cumulatively, copy number and expression of UT2 were significantly reduced in MM cell lines and primary MM patient cells, supporting our notion that the loss of UT2 is relevant to the pathogenesis of human myeloma.

The impact of UT2 on myeloma cell growth in vivo. To further explore the relationship of UT2 to myeloma biology, we examined whether the inverse effect of UT2 on myeloma cell growth in vitro (Figure $1 \mathrm{~F}$ ) affected an in vivo model of the disease. INA6 (Figure 4, A-C) or MM1S (Figure 4, D-F) myeloma cells with or without sgRNA-mediated depletion of UT2 were transplanted into recipient mice (60-63). As would be expected from the loss of a
STAT3 inhibitor, mice transplanted with sgRNA-mediated depletion of UT2 myeloma cells showed reduced disease survival compared with control recipient mice (Figure 4, A and D). Examining hematopoietic tissue by flow cytometry revealed increased myeloma cells in the BM (Figure 4, B and E, and Supplemental Figure 4, A and B). Animal mortality inversely correlated with levels of pSTAT3 $^{\mathrm{Y} 705}$ (Figure 4, C and F, and Supplemental Figure 4, A and B) and directly correlated with the change in UT2.

To investigate whether UT2 levels correlated with responsiveness of pSTAT $^{\mathrm{Y705}}$ or $\mathrm{PAKT}^{\mathrm{S473}}$ inhibition, we tested pharmacological inhibition of pSTAT3 ${ }^{\mathrm{y} 705}$ by using JAK inhibitors (AZD1480, refs. 64-68; and ruxolitinib, refs. 69-71) in INA6 cells with or without UT2 sgRNA (Figure 5, A and B, and Supplemental Figure $4 C)$. INA6 cell pSTAT3 ${ }^{\mathrm{Y} 705}$ levels were increased when UT2 was depleted by UT2 sgRNA. This increase was completely abrogated in the presence of AZD1480 or Ruxolitinib in INA6 cells with or without UT2 sgRNA. Similar results were seen in MM1S cells examining $\mathrm{pAKT}^{\mathrm{S} 473}$ levels by using the AKT inhibitors (perifosine, refs. 72-76; and MK2206, refs. 77-79) (Figure 5, D and E, and Supplemental Figure 4D). Conversely, we examined the effects of constitutive activation of STAT3 (STAT3C, refs. 80-84) or AKT (myristoylated AKT [myr-AKT], refs. 85, 86) in INA6 or MM1S cells expressing UT2. pSTAT3 ${ }^{\mathrm{Y} 705}$ (Figure 5C and Supplemental Figure $4 \mathrm{C}$ ) or $\mathrm{pAKT}^{\mathrm{S473}}$ (Figure $5 \mathrm{~F}$ and Supplemental Figure $4 \mathrm{D})$ were increased in these cells with constitutive expression of STAT3C or myr-AKT, respectively. The increases were abrogated by the overexpression of UT2. These data indicate that UT2 can serve to reduce activation events downstream of the binding partners we have defined for it: GP130 and RICTOR. Together, these results demonstrate that UT2 is capable of affecting the outcome of myeloma growth in association with changes in pSTAT3 ${ }^{\mathrm{Y} 705}$ expression. UT2 impairs STAT3 activation and, in so doing, influences the in vitro and in vivo growth of MM cells.

\section{Discussion}

In this study, we demonstrate that the transmembrane protein, UT2, functions to downmodulate STAT3 signaling. It directly interacts with GP130, resulting in specific reduction of pSTAT3 ${ }^{\mathrm{Y705}}$ (Figure 5G).

The interaction of UT2 with GP130 occurs independently of IL6R and of STAT3 itself, as indicated by the in vitro translation experiments. Therefore, it does have specificity of interaction that then affects the ability of heterologous components of a signal transduction module to form a functional unit. This is similar to what was observed when investigating how UT2 affects mTORC2 phosphorylation of $\mathrm{pAKT}^{\mathrm{S} 473}$ (2). In that context, UT2 bound directly to RICTOR but not to other parts of the mTORC2 complex, nor did it bind to RAPTOR, which is the scaffold for mTORC1. In both cases, the cytoplasmic portion of UT2 was essential for its binding with heterologous partners, and binding was inhibitory of the downstream phosphorylation events and impacted hematopoietic cell numbers. Therefore, UT2, like GP130 itself (87), may bind multiple distinct molecular partners. In so doing, it appears to provide a suppressive effect on activation signaling. Whether UT2 may influence other aspects of STAT3 or AKT signaling remains possible, but this issue will be explored in further studies.

It is apparent that UT2 is molecularly and functionally distinct from the SOCS family of gene products. For example, it has 
A

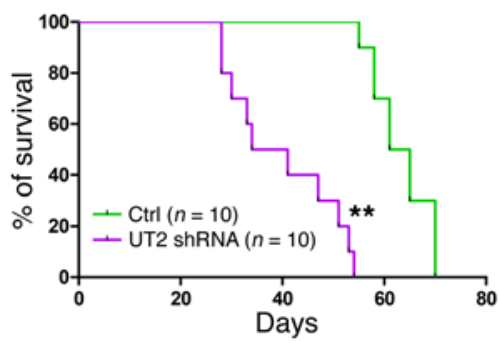

D

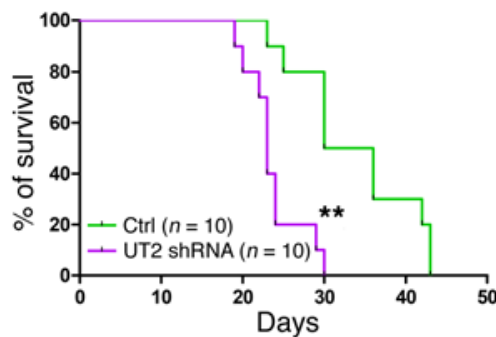

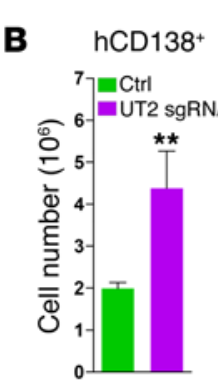

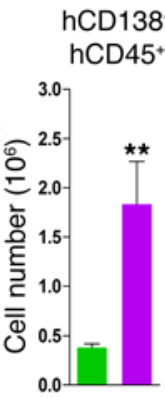

E

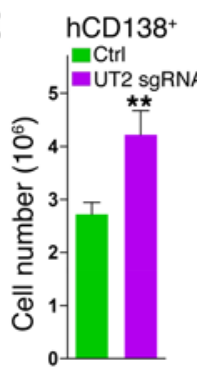

hCD138

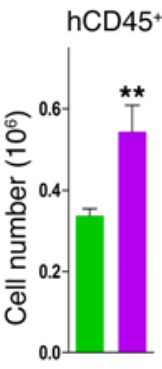

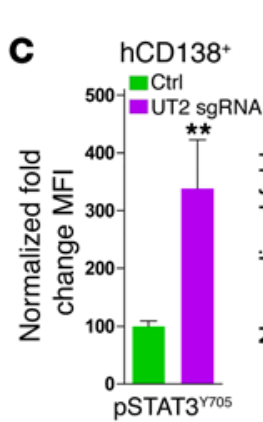
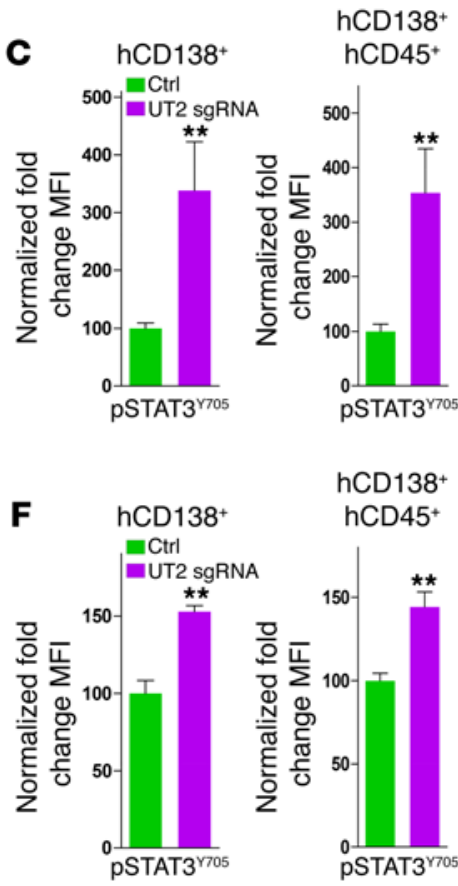

hCD138+ hCD45+

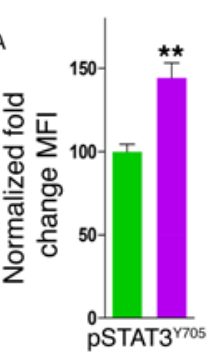

Figure 4. UT2 affects disease latency of myeloma in mice. (A) Kaplan-Meier survival curves for recipient mice after transplantation of sgRNA-mediated depletion of UT2 INA6 cells ( $n=10$ biological replicated experiments, log-rank test; ${ }^{*} P<0.01$ ). Animals were examined when moribund. (B) Flow cytometry analysis of hCD138 ${ }^{+}$and hCD138+hCD45+ BM cells from recipient mice in A. Data are shown as mean \pm SEM. ( $n=9-10$ biological replicated experiments, two-tailed, unpaired $t$ test; $\left.{ }^{* *} P<0.01\right)$. (C) Expression of pSTAT3 ${ }^{\mathrm{V} 705}$ in hCD138 ${ }^{+}$and hCD138 ${ }^{+}$hCD45 $5^{+}$BM cells from recipient mice in A. Data are shown as mean \pm SEM. ( $n=8-10$ biological replicated experiments, two-tailed, unpaired $t$ test; $\left.{ }^{* *} P<0.01\right)$. (D) Kaplan-Meier survival curves for recipient mice after transplantation of sgRNA-mediated depletion of UT2 MM1S cells ( $n=10$ biological replicated experiments, log-rank test; $\left.{ }^{* *} P<0.01\right)$. Animals were examined when moribund. (E) Flow cytometry analysis of hCD138 ${ }^{+}$and hCD138+hCD $45^{+}$BM cells from recipient mice in $\mathbf{D}$. Data are shown as mean \pm SEM ( $n$ = 9-10 biological replicated experiments, two-tailed, unpaired $t$ test; ${ }^{* *} P<0.01$ ). (F) Expression of pSTAT3 ${ }^{\mathrm{r} 705}$ in hCD138 ${ }^{+}$and hCD138+hCD45+ BM cells from recipient mice in (D). Data are shown as mean \pm SEM $\left(n=8-10\right.$ biological replicated experiments, two-tailed, unpaired $t$ test; $\left.{ }^{* *} P<0.01\right)$.

no SH2 domain, and SH2 domains are critical for SOCS protein actions. It also does not have a 40 residue $C$ terminal SOCS box characteristic of SOCS proteins (20). Furthermore, UT2 is a transmembrane protein, a feature not seen in the SOCS proteins. In addition, we have shown that UT2 binds directly to GP130, while SOCS3 binding to GP130 depends on tyrosine phosphorylation. Therefore, UT2 is not a new member of the SOCS family.

In functional terms, SOCS proteins are downstream of JAK/ STAT signaling and are transactivated in response to activation of that pathway. They thereby serve in a classic negative-feedback loop. We hypothesize that UT2 is providing a distinctive mode of modulating the JAK/STAT pathway but that, like SOCS proteins, it serves a role in constraining cell activation through GP130. We propose that UT2 is upstream of STAT3/SOCS3 activation, serving to modulate signaling in advance of the reactive negative regulation imposed by SOCS induction by pSTAT. These distinct routes and/or processes for regulating STAT3 activation and the different localization of the molecules suggest unique functions in hematopoietic cell biology that are still to be elucidated.

Our analyses of patient data indicate that UT2 is deleted or downregulated in myeloma cells and is inversely correlated with pSTAT3 levels in MM patients. Given the impact of UT2 as an inhibitor of myeloma lethality in vivo, as demonstrated by our CRISPR/Cas9 studies, we suggest that downregulating or deleting UT2 is an advantage to plasma cells. We therefore propose UT2 as a key, previously unrecognized participant in the pathophysiologic process of MM. It points to an additional molecular contributor to that process that may provide new pharmacologic targeting opportunities. Furthermore, it offers an additional model for how cell-activating signals can be checked at the cell surface, and while we do not know if UT2 itself functions as a receptor, the presence of its external domain suggests that external cues may be differentially affected by its presence.

\section{Methods}

Mice and animal procedures. All mice were kept in a specific pathogenfree facility at Massachusetts General Hospital. All mice studies and breeding were carried out under the approval of Institutional Animal Care and Use Committee of Massachusetts General Hospital. sgRNAmediated depletion of UT2 MM1S and INA6 cells $\left(5 \times 10^{6}\right)$ were transplanted into NOD/SCID mice (The Jackson Laboratory) that had been previously irradiated with 2 Gy (60-63). Animals were monitored daily and, when morbid, were euthanized.

MM patient samples. BM aspirates from MM patients were collected under a protocol approved by the IRB (13-583) of Massachusetts General Hospital. Ficoll density gradient was then used to recover viable mononuclear cells from BM aspirates. The patients were diagnosed with MM at a median age of 72, ranging from 56-91 years. Diagnosis was based on the International Myeloma Working Group criteria (88). All the MM patients presented with active MM and relapsed-refractory disease. 

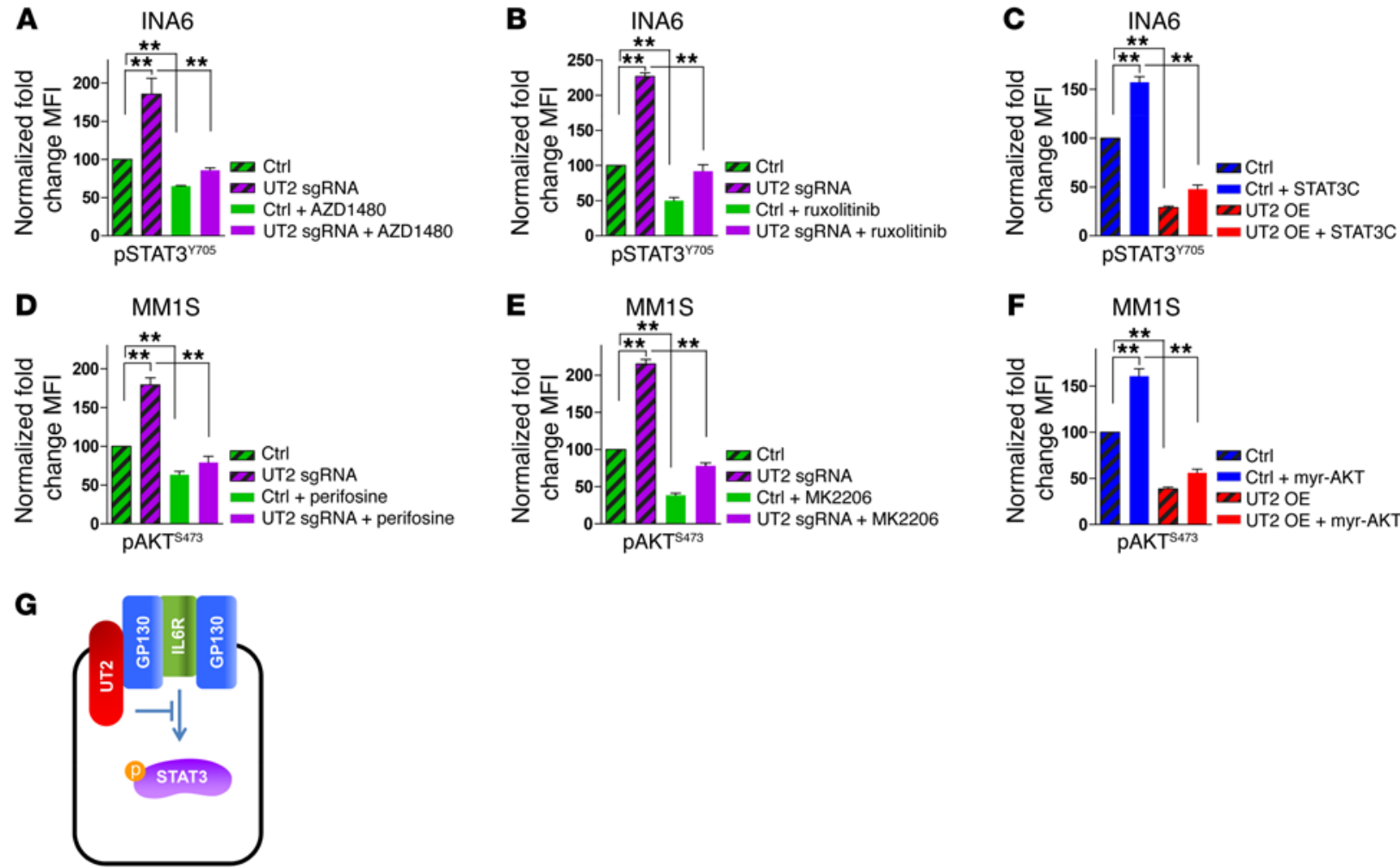

Figure 5. Effects of UT2 on pSTAT3 ${ }^{\text {v705 }}$ or pAKT ${ }^{5473}$ pharmacologic inhibition in myeloma cells. (A-C) Flow cytometry was performed on sgRNA-mediated depletion of UT2 INA6 cells with treatment of AZD1480 (A) or ruxolitinib (B), or overexpressing (OE) UT2 INA6 cells with STAT3C expression (C). sgRNAmediated depletion of UT2 INA6 cells were cultured with AZD1480 $(10 \mu \mathrm{M}, 6 \mathrm{~h})$ or ruxolitinib $(10 \mu \mathrm{M}, 2 \mathrm{~h})$, respectively. Quantification shown of the normalized fold-change in MFI for the indicated expression of pSTAT3 ${ }^{\mathrm{V705}}$ in these cells. Data are shown as mean \pm SEM ( $n=3-4$ experiments; two-tailed, unpaired $t$ test; ${ }^{* *} P<0.01$ ). See also Supplemental Figure 4C. (D-F) Flow cytometry was performed on sgRNA-mediated depletion of UT2 MM1S cells with treatment of perifosine (D) or MK2206 (E), or OE UT2 MM1S cells with myr-AKT expression (F). sgRNA-mediated depletion of UT2 MM1S cells were cultured with perifosine $(10 \mu \mathrm{M}, 6 \mathrm{~h})$ or MK2206 $(10 \mu \mathrm{M}, 2 \mathrm{~h})$, respectively. Quantification shown of the normalized fold change in MFI for the indicated expression of pAKT ${ }^{5473}$ in these cells. Data are shown as mean \pm SEM ( $n=3-4$ experiments; two-tailed, unpaired $t$ test; $\left.{ }^{* *} P<0.01\right)$. See also Supplemental Figure 4D. (C) Model of UT2 negative regulation of STAT3 function via modulating GP130 in myeloma cells.

CRISPR-Cas9 system and sgRNA design. The lentiCRISPR vector was purchased from Addgene. To construct the lentiviral sgRNA Cas9 vector, sgRNAs were cloned into lentiCRISPR vector in the BsmBI site (36). Lentivirus production and purification were performed as previously described (2). sgRNAs were designed using the CRISPR tool $(36,37)$ (http://crispr.mit.edu) to minimize potential off-target effects. The lentiCRISPR with sgRNAs targeting UT2 were cloned using the following sequences: UT2 sgRNA1: GTCATCGGTGTTCATCTTAT; UT2 sgRNA2: GCACATGTTCATGCGGAAAA; UT2 sgRNA3: CAGCGTTGCCACACAATGTC; UT2 sgRNA4: CTCAAAGCAGACTCCCCAAC; Ut2 sgRNA1: ATGCTCCAGGACTCAATTAC; Ut2 sgRNA2: TTGGTCTGCTCATAGTGGAT; Ut2 sgRNA3: GTTGGTCTGCTCATAGTGGA; and Ut2 sgRNA4: CAATTCCAGTAATTGAGTCC.

Materials and cell culture. STAT3, GP130, and IL6R plasmids were purchased from OriGene. Antibodies to AKT (catalog 4691), pAKT $^{\mathrm{s} 73}$ (catalog 4060), STAT3 (catalog 4904), pSTAT3 ${ }^{\mathrm{Y705}}$ (catalog 9145), pSTAT1 $^{\mathrm{Y} 701}$ (catalog 9167), pSTAT5 ${ }^{\mathrm{Y} 694}$ (catalog 4322), MYC (catalog 2278), and ACTIN (catalog 4970) were from Cell Signaling Technology. GP130 (catalogs sc655 and sc656) and IL6R (catalogs sc661 and sc13947) were from Santa Cruz Biotechnology Inc. FLAG M2 antibody (catalog F1804) was from Sigma-Aldrich. Antibodies to GFP (catalog ab290), MYC (catalog ab32), and ACTIN (catalog ab3280) were from Abcam. AZD1480 (catalog S2162) (64-68), ruxolitinib (catalog S1378) (69-71), perifosine (catalog S1037) (72-76), and MK2206 (catalog S1078) (77-79) were purchased from Selleckchem. HEK293T, MDA-MB231, T47D, DU145, and PC3 cells were purchased from ATCC. MM cell lines (INA6, MM1S, H929, and U266) were gifts from N. Raje (Cancer Center, Massachusetts General Hospital, Boston, Massachusetts, USA). MDA1833 and A395 cells were gifts from N. Baryawno (Center for Regenerative Medicine, Massachusetts General Hospital, Boston, Massachusetts, USA). IL6-dependent cell line INA6 was maintained in medium supplemented with $2.5 \mathrm{ng} / \mathrm{ml}$ IL6 (R\&D Systems). Cell proliferation of sgRNA-mediated depletion of UT2 or overexpressing UT2 INA6 cells were followed by cell counting of samples in quadruplicate using a cellometer (Nexcelom Bioscience). For IL6-stimulation/withdrawal experiments, sgRNA-mediated depletion of UT2 or overexpressing UT2 INA6 and HEK293T cells were deprived of serum for 24 hours. Then, IL6 (20 $\mathrm{ng} / \mathrm{ml}$ ) was added for 30 minutes (89).

Protein analyses. Western blot analysis was carried out according to standard methods as previously described (2). For IP experiments, cells were lysed with Triton X-100 containing lysis buffer as previously described (2). Data consisted of quantities with the Odyssey Infrared Imaging System (LI-COR Biosciences). 
Quantitative PCR ( $q P C R)$ and reverse-transcriptase PCR (RT-PCR). Reverse transcription PCR (RT-PCR) and quantitative PCR (qPCR) were performed as previously described $(2,90,91)$.

Flow cytometry. Phosphoflow experiments were as previously described $(2,92,93)$. Stained cells were analyzed with an LSRII, FACSCalibur, and Accuri flow cytometer (BD Biosciences). Cell sorting was performed with a FACSAriaII instrument (BD Biosciences). Data acquisition and analysis were performed with Cell Quest Pro or Diva software (BD Biosciences) and with FlowJo software (Tree Star Inc.), respectively.

In silico analysis of UT2 copy number and expression in hematologic tumors. Kaplan-Meier survival curves for UT2 were obtained using the tools at http://r2.amc.nl based on the GSE2658 data set (58) and probe set 1557176_a_at. Individuals with MM patients were subdivided based on median UT2 expression levels (high expression $[n=160]$ and low expression $[n=382]$ ) in tumor cells. Comparative analyses between normal cells and tumor cells were conducted with the use of publicly available microarray data from the Oncomine database (94). To relate UT2 copy number, the Broad-Novartis Cancer Cell Line Encyclopedia ( $n=338)$, GSE20306 data set $(n=449)$, GSE26849 data set $(n=261)$, and GSE15695 data set $(n=168)$ were used. To relate UT2 expression levels, the GSE2658 ( $n=414 \mathrm{CD} 138^{+}$purified BM samples from patients with MM), GSE4452 ( $n=65 \mathrm{MM}$ samples from patients with MM), GSE15695 ( $n=247$ CD138 ${ }^{+}$purified MM samples from patients with MM), GSE26790 ( $n=304 \mathrm{CD}^{2} 38^{+}$purified MM samples from patients with MM), GSE29023 ( $n=115)$, GSE7186 $(n=98)$, GSE2223 $(n=54)$, GSE30195 ( $n=19), \operatorname{GSE} 15061$ ( $n=202)$, GSE13159 $(n=542)$, GSE3678 $(n=14), \operatorname{GSE} 4290(n=153)$, and GSE6791 $(n=28)$ data sets were used.

Statistics. Sample size required for the experiments was estimated based on results of preliminary data. In vitro and in vivo data were analyzed with a two-tailed, unpaired Student's $t$ test (GraphPad Prism [GraphPad Software Inc.] and SigmaPlot software [SPSS Inc.]). $P$ val- ues less than 0.05 were considered statistically significant $\left({ }^{* *} P<0.01\right)$. The Kaplan-Meier log-rank test was used to analyze mouse survival data using GraphPad Prism. No blinding or randomization was performed for any of the experiments.

Study approval. All mice were kept in a specific pathogen-free facility at Massachusetts General Hospital. All mice studies and breeding were carried out under the approval of Institutional Animal Care and Use Committee of Massachusetts General Hospital. BM aspirates from MM patients were collected under a protocol approved by the IRB (13-583) of Massachusetts General Hospital.

\section{Author contributions}

DL and DTS designed research, analyzed data, and wrote the paper. DL carried out most of the experimental work with the help of YHW, DK, JR, HE, DBS, NR, and DTS directed the research.

\section{Acknowledgments}

We thank those who funded this work: NIH NHLBIU01HL100402, HL97794, HL044851, and the Ellison Medical Foundation (to D. Lee and D.T. Scadden); MGH Fund for Medical Discovery fellowship (D. Lee); and the MGH/HSCI CRM flow cytometry core (L. Prickett, A. Galvin, M. Handley, and M. Weglarz). We thank Scadden lab members for advice and helpful comments and $\mathrm{IH} \mathrm{Oh}$ (Catholic High Performance Cell Therapy Center, Catholic University, Seoul, South Korea) for MIG-STAT3C plasmid. The data reported in this manuscript are tabulated in the main paper and in the supplementary materials.

Address correspondence to: David T. Scadden, 185 Cambridge Street, Boston, Massachusetts 02114, USA. Phone: 617.726.5615; E-mail: david_scadden@harvard.edu.
1. Raaijmakers MH, et al. Bone progenitor dysfunction induces myelodysplasia and secondary leukaemia. Nature. 2010;464(7290):852-857.

2. Lee D, et al. Transmembrane inhibitor of RICTOR/mTORC2 in hematopoietic progenitors. Stem Cell Reports. 2014;3(5):832-840.

3. Levy DE, Darnell JE. Stats: transcriptional control and biological impact. Nat Rev Mol Cell Biol. 2002;3(9):651-662.

4. Bowman T, Garcia R, Turkson J, Jove R. STATs in oncogenesis. Oncogene. 2000;19(21):2474-2488.

5. Aaronson DS, Horvath CM. A road map for those who don't know JAK-STAT. Science. 2002;296(5573):1653-1655.

6. Yu H, Jove R. The STATs of cancer - new molecular targets come of age. Nat Rev Cancer. 2004;4(2):97-105.

7. Frank DA. STAT3 as a central mediator of neoplastic cellular transformation. Cancer Lett. 2007;251(2):199-210.

8. Benekli M, et al. Constitutive activity of signal transducer and activator of transcription 3 protein in acute myeloid leukemia blasts is associated with short disease-free survival. Blood. 2002;99(1):252-257.

9. Selander KS, et al. Inhibition of gp130 signaling in breast cancer blocks constitutive activation of Stat 3 and inhibits in vivo malignancy. Cancer Res. 2004;64(19):6924-6933.
10. Takeda K, Kaisho T, Yoshida N, Takeda J, Kishimoto T, Akira S. Stat3 activation is responsible for IL-6-dependent T cell proliferation through preventing apoptosis: generation and characterization of T cell-specific Stat3-deficient mice. JImmunol. 1998;161(9):4652-4660.

11. Lee CK, et al. STAT3 is a negative regulator of granulopoiesis but is not required for G-CSF-dependent differentiation. Immunity. 2002;17(1):63-72.

12. Welte T, et al. STAT3 deletion during hematopoiesis causes Crohn's disease-like pathogenesis and lethality: a critical role of STAT3 in innate immunity. Proc Natl Acad Sci US A. 2003;100(4):1879-1884.

13. Jenkins BJ, Roberts AW, Najdovska M, Grail D, Ernst M. The threshold of gp130-dependent STAT3 signaling is critical for normal regulation of hematopoiesis. Blood. 2005;105(9):3512-3520.

14. Jenkins BJ, et al. Pathologic consequences of STAT3 hyperactivation by IL- 6 and IL-11 during hematopoiesis and lymphopoiesis. Blood. 2007;109(6):2380-2388.

15. Aggarwal BB, et al. Signal transducer and activator of transcription-3, inflammation, and cancer: how intimate is the relationship? Ann N Y Acad Sci. 2009;1171:59-76.

16. Taga $\mathrm{T}$, et al. Interleukin- 6 triggers the association of its receptor with a possible signal trans- ducer, gp130. Cell. 1989;58(3):573-581.

17. Monaghan KA, Khong T, Burns CJ, Spencer A. The novel JAK inhibitor CYT387 suppresses multiple signalling pathways, prevents proliferation and induces apoptosis in phenotypically diverse myeloma cells. Leukemia. 2011;25(12):1891-1899.

18. Stahl N, Farruggella TJ, Boulton TG, Zhong Z, Darnell JE, Yancopoulos GD. Choice of STATs and other substrates specified by modular tyrosine-based motifs in cytokine receptors. Science. 1995;267(5202):1349-1353.

19. Abe K, et al. The YXXQ motif in gp 130 is crucial for STAT3 phosphorylation at Ser727 through an $\mathrm{H} 7$-sensitive kinase pathway. Oncogene. 2001;20(27):3464-3474.

20. Alexander WS. Suppressors of cytokine signalling (SOCS) in the immune system. Nat Rev Immunol. 2002;2(6):410-416.

21. Schmitz J, Weissenbach M, Haan S, Heinrich PC, Schaper F. SOCS3 exerts its inhibitory function on interleukin-6 signal transduction through the SHP2 recruitment site of gp130. J Biol Chem. 2000;275(17):12848-12856.

22. Krebs DL, Hilton DJ. SOCS proteins: negative regulators of cytokine signaling. Stem Cells. 2001;19(5):378-387.

23. Schuringa JJ, Wierenga AT, Kruijer W, Vellenga E. Constitutive Stat3, Tyr705, and Ser727 phosphorylation in acute myeloid leukemia cells caused 
by the autocrine secretion of interleukin-6. Blood. 2000;95(12):3765-3770.

24. Brender C, et al. STAT3-mediated constitutive expression of SOCS-3 in cutaneous T-cell lymphoma. Blood. 2001;97(4):1056-1062.

25. Sakai I, Takeuchi K, Yamauchi H, Narumi H, Fujita S. Constitutive expression of SOCS3 confers resistance to IFN- $\alpha$ in chronic myelogenous leukemia cells. Blood. 2002;100(8):2926-2931.

26. Cho-Vega JH, et al. Suppressor of cytokine signaling 3 expression in anaplastic large cell lymphoma. Leukemia. 2004;18(11):1872-1878.

27. Dechow T, et al. GP130 activation induces myeloma and collaborates with MYC. JClin Invest. 2014;124(12):5263-5274.

28. Brown R, et al. Phospho-flow detection of constitutive and cytokine-induced pSTAT3/5, pAKT and pERK expression highlights novel prognostic biomarkers for patients with multiple myeloma. Leukemia. 2015;29(2):483-490.

29. Hideshima T, Mitsiades C, Tonon G, Richardson PG, Anderson KC. Understanding multiple myeloma pathogenesis in the bone marrow to identify new therapeutic targets. Nat Rev Cancer. 2007;7(8):585-598.

30. Buerger C, et al. Sequence-specific peptide aptamers, interacting with the intracellular domain of the epidermal growth factor receptor, interfere with Stat3 activation and inhibit the growth of tumor cells. J Biol Chem. 2003;278(39):37610-37621.

31. Song H, Wang R, Wang S, Lin J. A low-molecular-weight compound discovered through virtual database screening inhibits Stat3 function in breast cancer cells. Proc Natl Acad Sci USA. 2005;102(13):4700-4705.

32. Germain D, Frank DA. Targeting the cytoplasmic and nuclear functions of signal transducers and activators of transcription 3 for cancer therapy. Clin Cancer Res. 2007;13(19):5665-5669.

33. Siddiquee K, et al. Selective chemical probe inhibitor of Stat3, identified through structure-based virtual screening, induces antitumor activity. Proc Natl Acad Sci USA. 2007;104(18):7391-7396.

34. Ahmad R, Raina D, Meyer C, Kufe D. Triterpenoid CDDO-methyl ester inhibits the Janusactivated kinase-1 (JAK1) $\rightarrow$ signal transducer and activator of transcription-3 (STAT3) pathway by direct inhibition of JAK1 and STAT3. Cancer Res. 2008;68(8):2920-2926.

35. Hayakawa F, et al. A novel STAT inhibitor, OPB31121, has a significant antitumor effect on leukemia with STAT-addictive oncokinases. Blood Cancer J. 2013;3:e166.

36. Shalem O, et al. Genome-scale CRISPR-Cas9 knockout screening in human cells. Science. 2014;343(6166):84-87.

37. Wang T, Wei JJ, Sabatini DM, Lander ES. Genetic screens in human cells using the CRISPR-Cas9 system. Science. 2014;343(6166):80-84.

38. Ahmed ST, Ivashkiv LB. Inhibition of IL-6 and IL-10 signaling and Stat activation by inflammatory and stress pathways. JImmunol. 2000;165(9):5227-5237.

39. Kopantzev Y, Heller M, Swaminathan N, Rudikoff S. IL-6 mediated activation of STAT3 bypasses Janus kinases in terminally differentiated B lineage cells. Oncogene. 2002;21(44):6791-6800.
40. Sengupta TK, Talbot ES, Scherle PA, Ivashkiv LB. Rapid inhibition of interleukin-6 signaling and Stat 3 activation mediated by mitogen-activated protein kinases. Proc Natl Acad Sci U S A. 1998;95(19):11107-11112.

41. Sotiropoulos A, et al. Differential activation of Stat 3 and Stat 5 by distinct regions of the growth hormone receptor. Mol Endocrinol. 1996;10(8):998-1009.

42. von Laue $S$, et al. Stimulation of endogenous GH and interleukin- 6 receptors selectively activates different Jaks and Stats, with a Stat 5 specific synergistic effect of dexamethasone. JEndocrinol. 2000;165(2):301-311.

43. Wiejak J, Dunlop J, Mackay SP, Yarwood SJ. Flavanoids induce expression of the suppressor of cytokine signalling 3 (SOCS3) gene and suppress IL-6-activated signal transducer and activator of transcription 3 (STAT3) activation in vascular endothelial cells. Biochem J. 2013;454(2):283-293.

44. Winston LA, Hunter T. JAK2, Ras, and Raf are required for activation of extracellular signal-regulated kinase/mitogen-activated protein kinase by growth hormone. J Biol Chem. 1995;270(52):30837-30840.

45. Maritano D, et al. The STAT3 isoforms alpha and beta have unique and specific functions. Nat Immunol. 2004;5(4):401-409.

46. Rothenberg SM, et al. A genome-wide screen for microdeletions reveals disruption of polarity complex genes in diverse human cancers. Cancer Res. 2010;70(6):2158-2164.

47. Bredel M, et al. Functional network analysis reveals extended gliomagenesis pathway maps and three novel MYC-interacting genes in human gliomas. Cancer Res. 2005;65(19):8679-8689.

48. Reyes I, Geliebter J. PTC versus paired normal thyroid tissue. NCBI Web site. http://www.ncbi. nlm.nih.gov/geo/query/acc.cgi?acc=GSE3678. Updated December 23, 2105. Accessed January 20, 2016.

49. Sun L, et al. Neuronal and glioma-derived stem cell factor induces angiogenesis within the brain. Cancer Cell. 2006;9(4):287-300.

50. Andersson A, et al. Microarray-based classification of a consecutive series of 121 childhood acute leukemias: prediction of leukemic and genetic subtype as well as of minimal residual disease status. Leukemia. 2007;21(6):1198-1203.

51. Pyeon D, et al. Fundamental differences in cell cycle deregulation in human papillomaviruspositive and human papillomavirus-negative head/neck and cervical cancers. Cancer Res. 2007;67(10):4605-4619.

52. Mills KI, et al. Microarray-based classifiers and prognosis models identify subgroups with distinct clinical outcomes and high risk of AML transformation of myelodysplastic syndrome. Blood.2009;114(5):1063-1072.

53. Haferlach T, et al. Clinical utility of microarray-based gene expression profiling in the diagnosis and subclassification of leukemia: report from the International Microarray Innovations in Leukemia Study Group. J Clin Oncol. 2010;28(15):2529-2537.

54. Graubert TA, et al. Recurrent mutations in the U2AF1 splicing factor in myelodysplastic syndromes. Nat Genet. 2012;44(1):53-57.
55. Dickens NJ, et al. Homozygous deletion mapping in myeloma samples identifies genes and an expression signature relevant to pathogenesis and outcome. Clin Cancer Res. 2010;16(6):1856-1864.

56. Chapman MA, et al. Initial genome sequencing and analysis of multiple myeloma. Nature. 2011;471(7339):467-472.

57. Carrasco DR, et al. High-resolution genomic profiles define distinct clinico-pathogenetic subgroups of multiple myeloma patients. Cancer Cell. 2006;9(4):313-325.

58. Zhan F, et al. The molecular classification of multiple myeloma. Blood. 2006;108(6):2020-2028.

59. Zhou Y, et al. Prediction of cytogenetic abnormalities with gene expression profiles. Blood. 2012;119(21):e148-e150.

60. Mani M, et al. BCL9 promotes tumor progression by conferring enhanced proliferative, metastatic, and angiogenic properties to cancer cells. Cancer Res. 2009;69(19):7577-7586.

61. Chauhan D, et al. A small molecule inhibitor of ubiquitin-specific protease-7 induces apoptosis in multiple myeloma cells and overcomes bortezomib resistance. Cancer Cell. 2012;22(3):345-358

62. Glavey SV, et al. The sialyltransferase ST3GAL6 influences homing and survival in multiple myeloma. Blood. 2014;124(11):1765-1776.

63. Zhang Y, et al. Pyk2 promotes tumor progression in multiple myeloma. Blood. 2014;124(17):2675-2686.

64. McFarland BC, et al. Therapeutic potential of AZD1480 for the treatment of human glioblastoma. Mol Cancer Ther. 2011;10(12):2384-2393.

65. Scuto A, et al. The novel JAK inhibitor AZD1480 blocks STAT3 and FGFR3 signaling, resulting in suppression of human myeloma cell growth and survival. Leukemia. 2011;25(3):538-550.

66. Derenzini E, et al. The JAK inhibitor AZD1480 regulates proliferation and immunity in Hodgkin lymphoma. Blood Cancer J. 2011;1(12):e46.

67. Hedvat M, et al. The JAK2 inhibitor AZD1480 potently blocks Stat 3 signaling and oncogenesis in solid tumors. Cancer Cell. 2009;16(6):487-497.

68. Xin H, et al. Antiangiogenic and antimetastatic activity of JAK inhibitor AZD1480. Cancer Res. 2011;71(21):6601-6610.

69. Quintas-Cardama A, et al. Preclinical characterization of the selective JAK1/2 inhibitor INCB018424: therapeutic implications for the treatment of myeloproliferative neoplasms. Blood. 2010;115(15):3109-3117.

70. Harrison C, et al. JAK inhibition with ruxolitinib versus best available therapy for myelofibrosis. N Engl JMed. 2012;366(9):787-798.

71. Verstovsek S, et al. A double-blind, placebo-controlled trial of ruxolitinib for myelofibrosis. N Engl J Med. 2012;366(9):799-807.

72. Kim E, et al. Phosphorylation of EZH2 activates STAT3 signaling via STAT3 methylation and promotes tumorigenicity of glioblastoma stem-like cells. Cancer Cell. 2013;23(6):839-852.

73. Hideshima T, et al. Perifosine, an oral bioactive novel alkylphospholipid, inhibits Akt and induces in vitro and in vivo cytotoxicity in human multiple myeloma cells. Blood. 2006;107(10):4053-4062.

74. Fu L, et al. Perifosine inhibits mammalian target of rapamycin signaling through facilitating degradation of major components in the 
mTOR axis and induces autophagy. Cancer Res. 2009;69(23):8967-8976.

75. Fei HR, Chen G, Wang JM, Wang FZ. Perifosine induces cell cycle arrest and apoptosis in human hepatocellular carcinoma cell lines by blockade of Akt phosphorylation. Cytotechnology. 2010;62(5):449-460.

76. Catley L, et al. Alkyl phospholipid perifosine induces myeloid hyperplasia in a murine myeloma model. Exp Hematol. 2007;35(7):1038-1046.

77. Cheng Y, et al. eEF-2 kinase dictates cross-talk between autophagy and apoptosis induced by Akt Inhibition, thereby modulating cytotoxicity of novel Akt inhibitor MK-2206. Cancer Res. 2011;71(7):2654-2663.

78. Hirai H, et al. MK-2206, an allosteric Akt inhibitor, enhances antitumor efficacy by standard chemotherapeutic agents or molecular targeted drugs in vitro and in vivo. Mol Cancer Ther. 2010;9(7):1956-1967.

79. Ramakrishnan V, et al. Anti-myeloma activity of Akt inhibition is linked to the activation status of PI3K/Akt and MEK/ERK pathway. PLoS One. 2012;7(11):e50005.

80. Bromberg JF, et al. Stat 3 as an oncogene. Cell. 1999;98(3):295-303.
81. Chung YJ, Park BB, Kang YJ, Kim TM, Eaves CJ, Oh IH. Unique effects of Stat 3 on the early phase of hematopoietic stem cell regeneration. Blood. 2006;108(4):1208-1215.

82. Takahashi K, Yamanaka S. Induction of pluripotent stem cells from mouse embryonic and adult fibroblast cultures by defined factors. Cell. 2006;126(4):663-676.

83. Wu L, Du H, Li Y, Qu P, Yan C. Signal transducer and activator of transcription 3 (Stat3C) promotes myeloid-derived suppressor cell expansion and immune suppression during lung tumorigenesis. Am J Pathol. 2011;179(4):2131-2141.

84. Yu C, et al. Targeting the intrinsic inflammatory pathway: honokiol exerts proapoptotic effects through STAT3 inhibition in transformed Barrett's cells. Am J Physiol Gastrointest Liver Physiol. 2012;303(5):G561-G569.

85. Kharas MG, et al. Constitutively active AKT depletes hematopoietic stem cells and induces leukemia in mice. Blood.2010;115(7):1406-1415.

86. Kohn AD, Summers SA, Birnbaum MJ, Roth RA. Expression of a constitutively active Akt Ser/Thr kinase in 3T3-L1 adipocytes stimulates glucose uptake and glucose transporter 4 translocation. JBiol Chem. 1996;271(49):31372-31378.
87. Taniguchi K, et al. A gp130-Src-YAP module links inflammation to epithelial regeneration. Nature. 2015;519(7541):57-62.

88. International Myeloma Working Group. Criteria for the classification of monoclonal gammopathies, multiple myeloma related disorders: a report of the International Myeloma Working Group. Br J Haematol. 2003;121(5):749-757.

89. Ahmad R, et al. MUC1-C oncoprotein promotes STAT3 activation in an autoinductive regulatory loop. Sci Signal. 2011;4(160):ra9.

90. Lee D, et al. ER71 acts downstream of BMP, Notch, Wnt signaling in blood vessel progenitor specification. Cell Stem Cell. 2008;2(5):497-507.

91. Lee D, Kim T, Lim DS. The Er71 is an important regulator of hematopoietic stem cells in adult mice. Stem Cells. 2011;29(3):539-548.

92. Sykes SM, et al. AKT/FOXO signaling enforces reversible differentiation blockade in myeloid leukemias. Cell. 2011;146(5):697-708.

93. Kalaitzidis D, et al. mTOR complex 1 plays critical roles in hematopoiesis Pten-loss-evoked leukemogenesis. Cell Stem Cell. 2012;11(3):429-439.

94. Rhodes DR, et al. ONCOMINE: a cancer microarray database and integrated data-mining platform. Neoplasia. 2004;6(1):1-6. 